\title{
Enhanced Fingerprint-Based Location Estimation System in Wireless LAN Environment*
}

\author{
Wilson M. Yeung, Jun Yang Zhou, and Joseph K. Ng \\ Department of Computer Science \\ Hong Kong Baptist University \\ Kowloon Tong, Hong Kong \\ \{wilson, jyzhou, jng @ @ comp.hkbu . edu.hk
}

\begin{abstract}
Received Signal Strength (RSS) is one of the most useful information used for location estimation in Wireless LAN (WLAN). Most of the proposed WLAN positioning systems obtain RSS from either the Access Point or from the Mobile Device, but there are few researches that make use of the RSS obtained from both Access Points and Mobile Devices to perform location estimation. In this paper, we propose a new WLAN positioning system which makes use of the RSS collected from both the Access Points and Mobile Device. Our experimental result shows that the performance of our system is enhanced more than $23 \%$, as compares to the traditional fingerprint-based WLAN positioning system which uses either RSS information obtained at Access Points or Mobile Device exclusively.
\end{abstract}

Keywords: Wireless LAN, Positioning, Location Estimation, Ubiquitous Computing.

\section{Introduction}

Mobile and wireless communication have become a crucial part of our daily life, thanks to the growth of the mobile and wireless technologies. As the cost of computing device decreases, many people own one or more computing devices for various usages, such as personal communication, office works, and entertainment. The advance in technologies and their applications have make Ubiquitous Computing possible and makes Ubiquitous Computing the new era of Computing. In particular, user's location is an important information for ubiquitous computing, as it enables location-aware services and therefore makes ubiquitous computing more practical and useful for daily life. That's why we need to explore, investigate and enhance positioning technologies for facilitating ubiquitous computing. Nowadays, we can easily obtain our location coordinates by the Global Positioning System (GPS). However, we can only receive GPS services in outdoor environment, as the GPS devices rely on signals from the satellite system surrounding the globe. Therefore many indoor positioning solutions [1] [2] [3] [4] based on radio frequency (RF) has been proposed in recent years. Among these previous works, Wi-Fi (IEEE 802.11) positioning become a hot topic for indoor positioning,

\footnotetext{
* This work is supported by the RGC Central Allocation from the Hong Kong SAR Government under the grant no. HKBU $1 / 05 \mathrm{C}$.
} 
as Wireless LAN (WLAN) has became a basic infrastructure of many buildings. Many WLAN positioning systems have been proposed in the literature, and most of them use Received Signal Strength (RSS) as input data for location estimation. However, previous works mainly focused on using the RSS obtained either at Access Point (AP) or the Mobile Device (MD). In other words, there are few researches that use RSS obtained from both AP and MD as input data for location estimation. In fact, the signal strength of the signal transmitted from the AP to MD (downlink) and the signal transmitted from MD to AP (uplink) can be different, this is true even both AP and MD have the same level of transmission power [5], i.e. asymmetrical signal strength [5] [6]. Therefore, we can actually treat the uplink and downlink RSS as two different source of information for location estimation.

In this paper, we present a new WLAN positioning system, which use both uplink and downlink RSS as input data to locate the user. Similar to the RADAR system by Bahl et. al [7] [8], our system is based on location fingerprint to perform location estimation. Experimental results had shown that our system can provide a more accurate location, as compares to the system using either uplink or downlink RSS exclusively.

The rest of the paper is organized as follows. In Section 2 we introduce the related works. Section 3 presents our system. In Section 4 we describe our test site and the experiment setup, and present the experiment results and performance evaluation. And finally, in Section 5] we provide a summary of our study and research findings.

\section{Related Work}

There are many related works on WLAN by using RSS for estimating a user's location. The RADAR system by Bahl et. al. [7] [8] is a well known WLAN positioning system, which is a location fingerprint system. The sampling process of this system is using a mobile device (laptop computer) to broadcast packets and then measure the signal strength at the access points (Pentium-based PCs), i.e. the uplink approach. The RSS values from different access points are then gathered to form a RSS tuple. Besides the RADAR system [7] [8], other fingerprint-based [9] [10] system are also proposed, where these systems use the downlink approach to sample a RSS tuple. The basic idea of a location fingerprint system is to find out the nearest neighbor in the signal space [8] [7] [11] [9] [10] by calculating the distance(usually, the Euclidean distance) between the location of fingerprints recorded in the system and the RSS tuples obtained in realtime. In order to create location fingerprints, the sampling process must be taken at different pre-defined location, and hence a group of RSS tuples is collected at each of these known locations. Each group of RSS tuples is then used to build a location fingerprint ( Say, by taking average value of RSS from each of the access points, i.e. the RADAR approach [7] [8] ). These location fingerprints are then saved into the database of the location estimation system. Later on, in the locating phase, real-time or online RSS tuples at unknown location will be obtained, and the location that matches the closest RSS tuple (i.e. the location fingerprint) stored in the database will be the user's location.

Besides location fingerprint system, there is probability-based system which uses another way to handle the RSS samples. Basically, the probability-based system uses the 
RSS samples to create probability distributions of signal strength for known locations. When a RSS tuple is obtained in real-time, it is matched to these probability distributions to find out the location with the highest probability. The HORUS system by Youssef et al. [12] [13] [14] [15], and the system by Xiang et al. [16] are probabilitybased WLAN positioning system, and both systems use the downlink approach to obtain the RSS tuples.

In fact, a few commercial products for Wi-Fi positioning can be found. Ekahua [17] performs positioning by collecting the RSS samples at the mobile device to build radio maps. And Cisco [18] states that their location tracking system measures the signal strength from the mobile device at the access points.

Rather than Wi-Fi base system, some other RF-based positioning systems [2] [3] [1] [4] have been proposed. MoteTrack system by Lorincz et at. [2] is one of the RFbased positioning systems. It uses a numbers of wireless sensor nodes, i.e. "Mote", to build a sensor network and to determine user's location by using the RSS information. In fact, many RF-based positioning systems used wireless node as a sensor. These nodes send and receive beacon packets and therefore obtain the RSS information. By gathering the RSS data from all these nodes, the location of a mobile node can be estimated. In this kind of sensor network environment, both the uplink and downlink RSS of a mobile node can be retrieved.

Previous work on WLAN positioning has mainly focused on using the RSS samples either collected at AP or MD, but not using RSS samples obtained from both side. Ganu et al. [5] has investigated and reported the existance and the characteristic of asymmetrical signal strength. However, no positioning approach based on asymmetrical signal strength is proposed in their studies. In our previous research [6], we proposed a positioning model based on asymmetrical signal strength and make use of both uplink and downlink RSS information. Since results are promising, this work is a further enhancement of our previous work as reported in [6].

\section{Proposed System}

Different from most of the previous works, our system considered the existance of asymmetrical signal strength, and therefore we used both uplink and downlink RSS as input data for location estimation.

\subsection{Asymmetrical Signal Strength}

Theoretically, the received signal strength (RSS) should be proportional to the transmission power of transmitter. Supposes the distance between the transmitter and receiver is fixed, then the RSS value measured at receiver should be higher if we increase the output power of the transmission; or the RSS value should be lower if the transmission power is reduced. However, Ganu et al. [5] have shown that even the AP and MD has the same level of transmission power, the downlink and uplink RSS value measured at the AP and MD can be different due to different design [5]. In fact, the transmission power of the AP and MD are seldom at the same level, due to some practical reasons. For an example, the AP needs to cover a larger area and therefore it needs higher transmission power; On the other hand, the MD needs to save the battery power and usually 
the transmission power is much smaller as compared to the AP [6]. As long as asymmetrical signal strength exists, our proposed system has considered and made use of this characteristic to enhance the performance of the location estimation system.

\subsection{Methodology}

In our proposed system, we have two sets of training data: $\left\{s_{i}^{(d)}\right\}$ and $\left\{s_{i}^{(u)}\right\}$, which represent two sets of RSS data collected at some known location $l_{i}$ by the downlink, and uplink approach, respectively, in the offline (training) phase. Meanwhile, two observed RSS tuples, $s_{a}$ and $s_{b}$, are obtained at a unknown location $\theta$ by the downlink, and uplink approach, respectively, in the online phase. $s_{i}^{(d)}, s_{i}^{(u)}, s_{a}$ and $s_{b}$ are $m \times 1$ vectors, where $m$ is the number of access points. By combining two downlink and uplink data source, we have a training data source: $\left\{\left\{s_{i}^{(d)}\right\},\left\{s_{i}^{(u)}\right\}, l_{i}\right\}_{i=1}^{n}$, where $n$ is the number of sample points. Our proposed system is to find out the unknown location $\theta$ based on $\left(s_{a}, s_{b}\right)$, obtained in real-time, and the training data source. And hence, we need a metric to pick up the one that best matches the observed signal strength.

Euclidean distance is a common measurement to describe the distance between two points in free space, which is widely used in real application. Mahalanobis distance can be thought of as an extension to the Euclidean distance, which takes into account the correlations of the data set. In fact, Mahalanobis distance is widely used in cluster analysis and other classification techniques. The composed distance is a metric to describe the distance between two points based on the merged data source, while the metric of probability is based on the Maximum Likelihood rule. We assume that the received signals follow a multiple normal distribution, then we choose the one that maximizes the probability as the solution.

We describe these criteria in detail as follows:

Euclidean Distance. The idea of Euclidean Distance is to compute the distance between the observed RSS tuple, $s=\left(s_{1}, s_{2}, \ldots, s_{m}\right)^{T}$, and the mean RSS tuple, $\overline{s^{*}}=$ $\left(\overline{s_{1}^{*}}, \overline{s_{2}^{*}}, \ldots, \overline{s_{m}^{*}}\right)^{T}$. The distance is defined as:

$$
d=\sqrt{\left(s-\overline{s^{*}}\right)^{T}\left(s-\overline{s^{*}}\right)}
$$

Then

$$
\begin{cases}d a_{i}=\sqrt{\left(s_{a}-\overline{s_{i}^{(d)}}\right)^{T}\left(s_{a}-\overline{s_{i}^{(d)}}\right)}, & \text { downlink } \\ d b_{i}=\sqrt{\left(s_{b}-\overline{s_{i}^{(u)}}\right)^{T}\left(s_{b}-\overline{s_{i}^{(u)}}\right)}, & \text { uplink }\end{cases}
$$

where $\overline{s_{i}^{(d)}}$ and $\overline{s_{i}^{(u)}}$ are the mean of the recorded RSS at point $i$ from downlink and uplink respectively.

The result location is the one that minimizes $d a_{i}$ or $d b_{i}$, as the RADAR [7] [8] does, i.e.:

$$
\theta=\left\{l_{i}: d a_{i}=\min \left\{d a_{j}, j=1, \ldots, n\right\} \text { or } d b_{i}=\min \left\{d b_{j}, j=1, \ldots, n\right\}\right\}
$$


Mahalanobis Distance. Mahalanobis distance differs from Euclidean distance in that it takes into account the correlations of the data set.

Formally, Mahalanobis distance is defined as:

$$
m d=\sqrt{\left(s_{a}-\overline{s^{*}}\right)^{T}\left(\Sigma^{*}\right)^{-1}\left(s_{a}-\overline{s^{*}}\right)}
$$

Then we have,

$$
\begin{cases}m d a_{i}=\sqrt{\left(s_{a}-\overline{s_{i}^{(d)}}\right)^{T}\left(\Sigma_{i}^{(d)}\right)^{-1}\left(s_{a}-\overline{s_{i}^{(d)}}\right)}, & \text { downlink } \\ m d b_{i}=\sqrt{\left(s_{b}-\overline{s_{i}^{(u)}}\right)^{T}\left(\Sigma_{i}^{(u)}\right)^{-1}\left(s_{b}-\overline{s_{i}^{(u)}}\right)}, & \text { uplink }\end{cases}
$$

where $\overline{s_{i}^{(d)}}$ and $\overline{s_{i}^{(u)}}$ are the mean of the recorded RSS and $\Sigma_{i}^{(d)}$ and $\Sigma_{i}^{(u)}$ are the covariance of the recorded RSS at point $i$ from downlink, and uplink respectively.

Suppose $\left\{s_{i j}^{(d)}\right\}_{j=1}^{m}$ and $\left\{s_{i j}^{(u)}\right\}_{j=1}^{m}$ are the signals received from downlink and uplink in location $l_{i}$, then $\overline{s_{i}^{(d)}}, \overline{s_{i}^{(u)}}, \Sigma_{i}^{(d)}$ and $\Sigma_{i}^{(u)}$ are defined as:

$$
\begin{gathered}
\begin{cases}\overline{s_{i}^{(d)}}=\frac{1}{m} \sum_{j=1}^{m} s_{i j}^{(d)}, & \text { downlink } \\
\overline{s_{i}^{(u)}}=\frac{1}{m} \sum_{j=1}^{m} s_{i j}^{(u)}, & \text { uplink }\end{cases} \\
\begin{cases}\Sigma_{i}^{(d)}=\frac{1}{m} \sum_{j=1}^{m}\left(s_{i j}^{(d)}-\overline{s_{i}^{(d)}}\right)\left(s_{i j}^{(d)}-\overline{s_{i}^{(d)}}\right)^{T}, & \text { downlink } \\
\Sigma_{i}^{(u)}=\frac{1}{m} \sum_{j=1}^{m}\left(s_{i j}^{(u)}-\overline{s_{i}^{(u)}}\right)\left(s_{i j}^{(u)}-\overline{s_{i}^{(u)}}\right)^{T}, & \text { uplink }\end{cases}
\end{gathered}
$$

where $\Sigma_{i}^{(d)}$ and $\Sigma_{i}^{(u)}$ describe the covariance of the downlink data and uplink data.

The solution, i.e. the estimated location, is the location $i$ has the minimum value of $m d a_{i}$ or $m d b_{i}$, that is:

$$
\theta=\left\{l_{i}: m d a_{i}=\min \left\{m d a_{j}, j=1, \ldots, n\right\} \text { or } m d b_{i}=\min \left\{m d b_{j}, j=1, \ldots, n\right\}\right\}
$$

Composed Distance. We have the downlink data and the uplink data, and we want to combine these two sets of data to find out a criteria to choose the best solution. One intuitive idea is the one that composes $d a_{i}$ and $d b_{i}$, where $d a_{i}$ and $d b_{i}$ are defined by eq. (2), i.e. $d_{i}=d a_{i} \times d b_{i}$, which is a metric defined in the merge data space. The solution is the location that has the minimum $d_{i}$. Namely,

$$
\theta=\left\{l_{i}: d_{i}=\min \left\{d_{j}, j=1, \ldots, n\right\}\right\}
$$

Probability. Assuming $s_{a}$ and $s_{b}$ follow multiple normal distributions and they are independent, then we have:

$$
\operatorname{prob}\left(s_{a} \mid l\right)=\frac{1}{(2 \pi)^{m / 2} \mid \Sigma^{(d) \mid 1 / 2}} \exp \left(-\frac{\left(s_{a}-\overline{s^{(d)}}\right)^{T}\left(\Sigma^{(d)}\right)^{-1}\left(s_{a}-\overline{s^{(d)}}\right)}{2}\right)
$$


where $\overline{s^{(d)}}$ is the mean of the recorded RSS and $\Sigma^{(d)}$ is the covariance at location $l$ from the downlink data. Similarly, we can apply the same formula on the uplink data, i.e.

$$
\operatorname{prob}\left(s_{b} \mid l\right)=\frac{1}{(2 \pi)^{m / 2}\left|\Sigma^{(u)}\right|^{1 / 2}} \exp \left(-\frac{\left(s_{b}-\overline{s^{(u)}}\right)^{T}\left(\Sigma^{(u)}\right)^{-1}\left(s_{b}-\overline{s^{(u)}}\right)}{2}\right)
$$

where $\overline{s^{(u)}}$ is the mean and $\Sigma^{(u)}$ is the covariance at location $l$ from the uplink data.

We define a likelihood function with $s_{a}$ and $s_{b}$ based on location $l_{i}$ :

$$
\operatorname{prob}\left(s_{a}, s_{b} \mid l_{i}\right)=\operatorname{prob}\left(s_{a} \mid l_{i}\right) \operatorname{prob}\left(s_{b} \mid l_{i}\right)
$$

That is,

$\operatorname{prob}\left(s_{a}, s_{b} \mid l_{i}\right)=\frac{1}{c} \exp \left(-\frac{\left(s_{a}-\overline{s_{i}^{(d)}}\right)^{T}\left(\Sigma_{i}^{(d)}\right)^{-1}\left(s_{a}-\overline{s_{i}^{(d)}}\right)+\left(s_{b}-\overline{s_{i}^{(u)}}\right)^{T}\left(\Sigma_{i}^{(u)}\right)^{-1}\left(s_{b}-\overline{s_{i}^{(u)}}\right)}{2}\right)$

where $c=(2 \pi)^{m} \cdot\left|\Sigma_{i}^{(d)}\right|^{1 / 2} \cdot\left|\Sigma_{i}^{(u)}\right|^{1 / 2}$ is independent with $s_{a}$ and $s_{b}$, and it depends on $l_{i}$.

The solution is the location whose $\overline{s_{i}^{(u)}}$ and $\overline{s_{i}^{(d)}}$ can maximizes the likelihood function, which is the Maximum Likelihood Estimator (MLE).

We set

$$
\left\{\begin{array}{lll}
m d a_{i}^{2}=\left(s_{a}-s_{i}^{(d)}\right)^{T}\left(\Sigma_{i}^{(d)}\right)^{-1}\left(s_{a}-s_{i}^{(d)}\right), & & \text { downlink } \\
m d b_{i}^{2}=\left(s_{b}-s_{i}^{(u)}\right)^{T}\left(\Sigma_{i}^{(u)}\right)^{-1}\left(s_{b}-s_{i}^{(u)}\right), & & \text { uplink }
\end{array}\right.
$$

Therefore the likelihood function can be rewritten as:

$$
\operatorname{prob}\left(s_{a}, s_{b} \mid l_{i}\right)=\frac{1}{(2 \pi)^{m} \cdot\left|\Sigma_{i}^{(d)}\right|{ }^{1 / 2} \cdot\left|\Sigma_{i}^{(u)}\right|^{1 / 2}} \exp \left(-\frac{m d a_{i}^{2}+m d b_{i}^{2}}{2}\right)
$$

Then the solution is

$$
\theta=\left\{l_{i}: \operatorname{prob}\left(s_{a}, s_{b} \mid l_{i}\right)=\max \left\{\operatorname{prob}\left(s_{a}, s_{b} \mid l_{j}\right), j=1, \ldots, n\right\}\right\}
$$

In our system, we implemented both the "Composed Distance" and the "Probability" approaches, since they can make use of both the uplink and downlink RSS data simultaneously. We will compare these results with the RADAR system and the one with Mahalanobis distance and investigate how much performance gain can be obtained by utilizing both the uplink and downlink RSS data for location estimation.

\section{Performance}

In this section, we present the experiment setup for testing our proposed system, the experimental result and analysis for our study. 


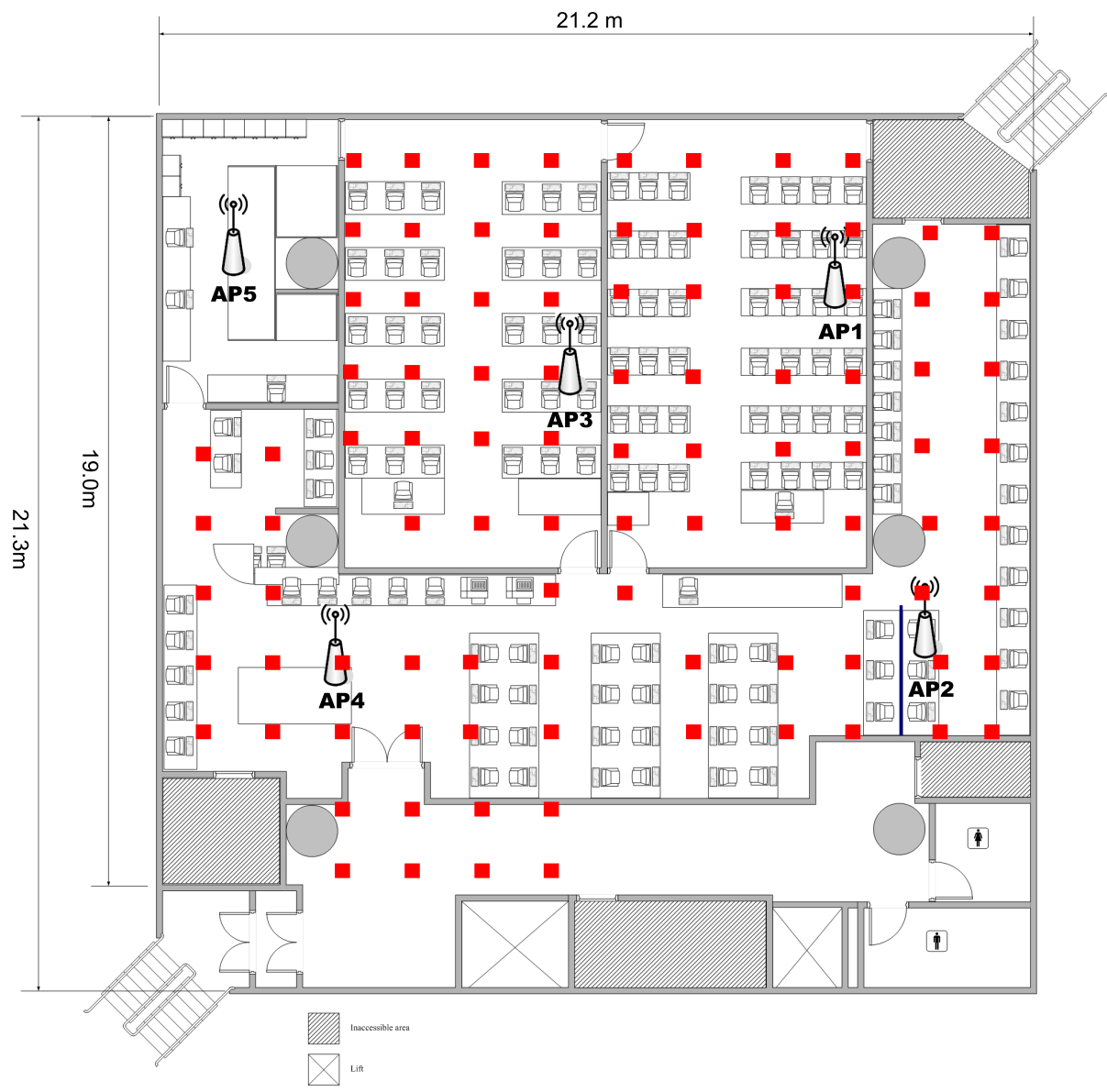

Fig. 1. The testbed for our experiment with 5 APs, where the red squares indicate the sampling locaions

\subsection{Experiment Setup}

Testbed for the experiment. Our experiments were performed on the third floor of a six-storey building, which is a computer laboratory of the Computer Science Department at HKBU. Figure 1 shows the floor plan of the lab and the dimension of the testbed are $21.3 \mathrm{~m}$ by $21.2 \mathrm{~m}$, covering an area of $451.56 \mathrm{~m}^{2}$.

In the testbed, five access points are installed. We used an open source wireless router (Linksys WRT54GL [19]) as our access point. Besides, we installed OpenWRT [20] to replace the original software that bundled with the router. OpenWRT is an open source Linux distribution, which allows us to have more control in sending and receiving signals to and from the mobile devices. To obtain RSS of the mobile device at the access point, we used a self-developed tool, which run on the access point, to grab the packets from the mobile device, and hence getting the RSS. All the RSS records were then sent 
to and stored in our database server. For the mobile device, we used the HP iPAQ 4150 Pocket PC, which has a built-in 802.11b wireless LAN card. We also developed another tool which runs on the Pocket PC in order to obtain the RSS sample at the mobile device. This tool can report the RSS from each access point by calling the system API to perform an active scan.

Data Collection. In the experiment, we identified 99 sampling locations in the public area of the testbed, marked as red square in floor plan of Figure 1 Each sampling location is about $1.8 \mathrm{~m}$ apart from the neighbor sampling locations. The area covered by the sampling location is about $305.5 \mathrm{~m}^{2}$. All sampling locations were covered by all 5 access points.

We collected RSS sample in each of the 4 directions ( north, east, south and west), and we collect 50 samples for each direction. We collected two sets of RSS samples, i.e. one set collected at the access point ( uplink approach ) and another set at the mobile device ( downlink approach). Each set of data contains 19,800 RSS samples. We used $80 \%$ of the samples as training data for training, and the rest of the $20 \%$ of the samples were used for testing. In each iteration of the test, we randomly selected one test sample from both sets of testing data, where both test samples are collected at same known location.

Performance Studies. In our experiments, we want to evaluate 1) the performance of a positioning system similar to RADAR system but using Mahalanobis Distance to replace Euclidean Distance( RADAR system uses Euclidean Distance ) on either uplink or downlink data( we use "System MD" to denote this system for the rest of the paper); 2) the performance of the system which applied "Composed Distance" or "Probability" methods where these methods use both uplink and downlink data. 3) The performance comparison between RADAR and our system, actually this is the comparison between using either uplink or downlink data and using both data.

\subsection{Experimental Results and Analysis}

Figure 2] 3 \& 4 show the the cumulative distribution function (CDF) of the error distance among different systems. From figure 2, we can observe that the accuracy of RADAR system and System MD are more or less the same when they were applied with the downlink data. System MD yielded better performance at $30 \%$ but it's worse than RADAR system at $85 \%$ of cumulatively probability. However, when the uplink data is applied, the two systems obtain different performance. Figure 3 shows that the the RADAR system performed better than System MD in most of the time. It is because the uplink signal is transmitted from MD to AP, and the transmission power of MD is smaller. If background noise in the testbed is constant, then uplink channel should has lower Signal to Noise Ratio (SNR), and therefore the uplink data should be more noisier than the downlink data. By comparing the experimental results of uplink and downlink data, we think that System MD is more sensitive to signal noise. The performance of our proposed system with "Composed Distance" and "Probability" are shown in figure 4 . In most of the time, the performance of these two approaches are the same, except the significant difference at $40 \%$ of cumulative probability. Among 


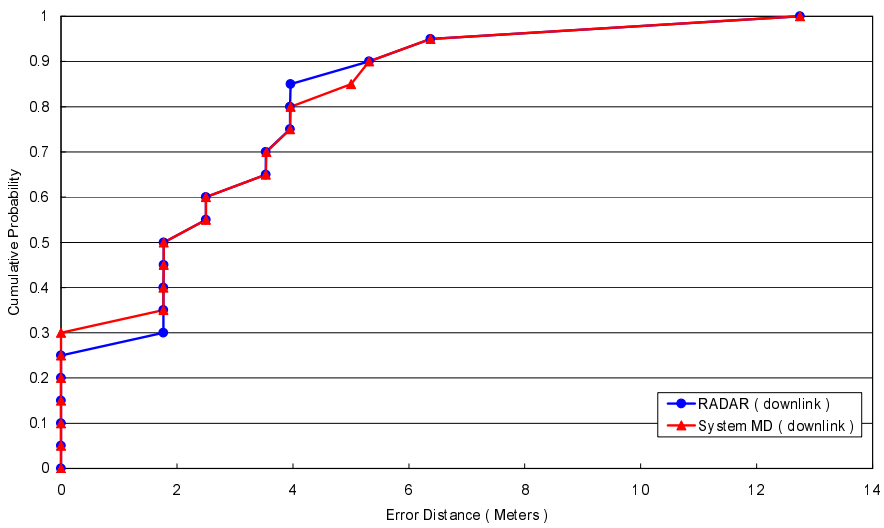

Fig. 2. Cumulative error distance of uplink data

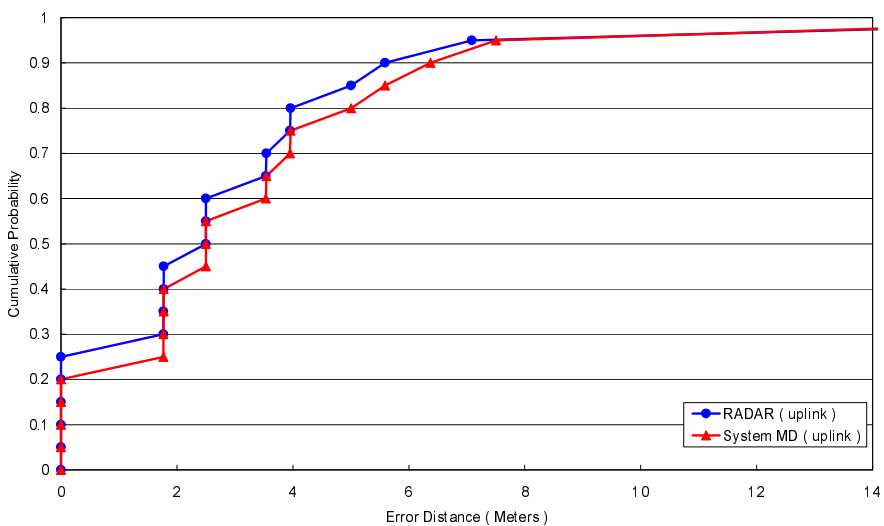

Fig. 3. Cumulative error distance of downlink data

Table 1. Performance Summary

\begin{tabular}{|c|c|c|c|c|c|c|}
\hline & \multicolumn{2}{|c|}{ RADAR } & \multicolumn{2}{c|}{ System MD } & \multicolumn{2}{c|}{ Our System } \\
\cline { 2 - 7 } & Downlink & Uplink & Downlink & Uplink & Mix( Composed Distance ) & Mix( Probability ) \\
\hline \hline Average $(\mathrm{m})$ & 2.47 & 2.65 & 2.42 & 2.97 & 1.88 & 1.78 \\
Std. Dev.(m) & 2.14 & 2.47 & 2.22 & 2.58 & 1.95 & 1.93 \\
Max $(\mathrm{m})$ & 12.75 & 21.30 & 12.75 & 20.16 & 18.81 & 13.82 \\
Min $(\mathrm{m})$ & 0.00 & 0.00 & 0.00 & 0.00 & 0.00 & 0.00 \\
$90 \%$ Percentile $(\mathrm{m})$ & 5.31 & 5.59 & 5.31 & 6.37 & 3.96 & 3.96 \\
Confidence Interval $(\alpha=0.05)(\mathrm{m})$ & 0.039 & 0.045 & 0.041 & 0.047 & 0.036 & 0.035 \\
\hline
\end{tabular}

these figures, we can obverse that the RADAR system and System MD perform better when they were applied with uplink data, as compared with result of the downlink data. 


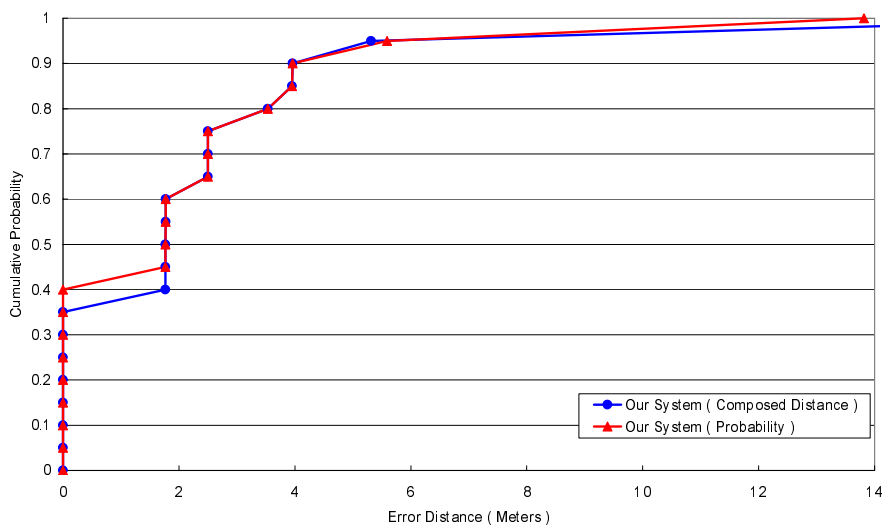

Fig. 4. Cumulative error distance of our system

Table 2. Enhancement in percentage

\begin{tabular}{c|c|c|}
\hline & \multicolumn{2}{|c|}{ Our System } \\
\cline { 2 - 3 } & Composed Distance Technique & Probability Technique \\
\hline \hline RADAR (downlink) & $23.90 \%$ & $28.98 \%$ \\
RADAR (uplink) & $27.78 \%$ & $32.60 \%$ \\
\hline
\end{tabular}

However, when both uplink and downlink data were applied, our proposed system performs better than systems using uplink data and, of course ,the downlink data.

Table 1 summarized the performance of RADAR, System MD, and our proposed system. Our proposed system yielded a smaller average error distance over the other two systems. The average error distance of the system with "Composed Distance" method is $1.88 \mathrm{~m}$ and, the system with the "Probability" method yield a slightly smaller value, i.e. $1.78 \mathrm{~m}$. System MD with uplink RSS data obtained the largest value of average error distance, that is $2.97 \mathrm{~m}$. The standard deviation of the error distance of the system with the "Probability" method is $1.93 \mathrm{~m}$, which is the smallest value among all systems. However, when the RADAR system and System MD used with downlink data, both of them obtained the smallest value for the Maximum error distance, i.e. $12.75 \mathrm{~m}$. Besides, the maximum error distance of the system with the "Probability" method is about $5 \mathrm{~m}$ shorter than the system with the "Composed Distance" method. Finally, Table 2 shows the performance enhancement of our system over the RADAR system. The performance of our system has our performed the RADAR system by $23.90 \%$ to $32.60 \%$.

In the point of view of accuracy, "Probability" methods are more accurate than "Composed Distance" methods, but it demands a higher computation cost. On the other hand, "Composed Distance" methods perform nearly the same as "Probability" methods but with a lower computation cost. Therefore if we demand better accuracy, we should adopt the "Probability" methods, otherwise, for lower computation cost, one should adopt the "Composed Distance" method for a comparable accuracy. 


\section{Summary and Future Work}

In this paper, we presented a WLAN positioning system which fully utilized the RSS collected from both the access points and mobile device. In our experiment, the accuracy of our system has improved for $23 \%$ to $32 \%$ over the traditional fingerprint-based positioning system.

Experimental results show that uplink data are noisy than the downlink data, and therefore the use of the uplink data may lower the performance of a positioning system. However, we can consider to apply other positioning algorithm, rather than location fingerprint algorithm, on the uplink data. In other words, we can actually apply two different positioning algorithms on downlink and uplink data, and this approach may further enhance the accuracy of user's position. We are going to investigate and evaluate this approach as our future work. In fact, if two different algorithms are applied, we may need a method to combine the results generated by the algorithms and, therefore, we will also investigate this kind of methods in our future research.

\section{References}

1. Ray, S., Starobinski, D., Trachtenberg, A., Ungrangsi, R.: Robust location detection with sensor networks

2. Lorincz, K., Welsh, M.: A robust, decentralized approach to rf-based location tracking. Technical Report TR-04-04, Harvard University (2004)

3. Ramadurai, V., Sichitiu, M.L.: Localization in wireless sensor networks: A probabilistic approach. In: ICWN 2003. Proceedings of the 2003 International Conference on Wireless Networks, pp. 275-281 (2003)

4. Vandenbussche, K.: Fine-grained indoor localization using wireless sensor nodes. Master's thesis, Delft University of Technology (August 2005)

5. Ganu, S., Krishnakumar, A.S., Krishnan, P.: Infrastructure-based location estimation in wlan networks. In: WCNC 2004. Proceedings of the IEEE Wireless Communications and Networking Conference (2004)

6. Yeung, W.M., Ng, J.K.: Wireless LAN Positioning based on Received Signal Strength from Mobile device and Access Points. In: Proceedings of RTCSA (to appear 2007)

7. Bahl, P., Padmanabhan, V.N.: RADAR: An in-building RF-based user location and tracking system. INFOCOM (2), 775-784 (2000)

8. Bahl, P., Balachandran, A., Padmanabhan, V.: Enhancements to the RADAR user location and tracking system. Technical report, Microsoft Corporation (February 2000)

9. Wong, W.H., Ng, J.K., Yeung, W.M.: Wireless lan positioning with mobile devices in a library environment. In: Proceedings of ICDCS-MDC 2005 Workshop, Columbus, Ohio, USA, pp. 633-636 (2005)

10. Yeung, W.M., Ng, J.K.: An Enhanced Wireless LAN Positioning Algorithm based on the Fingerprint Approach. In: Proceedings of IEEE TENCON 2006, Hong Kong, China (November 2006)

11. Cheng, Y., Chawathe, Y., LaMarca, A., Krumm, J.: Accuracy characterization for metropolitan-scale wi-fi localization (2005)

12. Youssef, M., Agrawala, A.: The horus wlan location determination system (June 2005)

13. Youssef, M., Agrawala, A., Shankar, U.: Wlan location determination via clustering and probability distributions (March 2003) 
14. Youssef, M., Agrawala, A.: On the optimality of wlan location determination systems. Technical Report UMIACS-TR 2003-29 and CS-TR 4459, University of Maryland, College Park (March 2003)

15. Youssef, M.A., Agrawala, A., Shankar, A.U., Noh, S.H.: A probabilistic clustering-based indoor location determination system. Technical Report UMIACS-TR 2002-30 and CS-TR 4350, Department of Computer Science and UMIACS, University of Maryland (March 2002)

16. Xiang, Z., Song, S., Chen, J., Wang, H., Huang, J., Gao, X.: A wireless lan-based indoor positioning technology. IBM Journal of Research and Development 48 (2004)

17. Ekahau, Inc., http: / / www . ekahau.com

18. Cisco Systems, Inc.: Wi-fi based real-time location tracking: Solutions and technology (2006)

19. Cisco Systems, Inc.: Linksys WRT54GL V1.1 Wireless-G Broadband Router (2006), http: / /www. linksys.com/

20. OpenWRT: White Russian RC6 (2006), http: / / openwrt.org/ 